\title{
The ResearchAbility initiative: Towards a more inclusive environment in research
}

\author{
Gian Maria Greco ${ }^{a, b,{ }^{*}}$ Alexandra Nothnagel ${ }^{a, c}$ and Carlo Antonini ${ }^{a, d}$ \\ ${ }^{a}$ Marie Curie Alumni Association, \\ Kunstlaan 24, Brussels, Belgium \\ ${ }^{b}$ Institute of Applied Linguistics, University of Warsaw \\ Dobra 55, Warsaw, Poland \\ ${ }^{c}$ Atos International \\ River Ouest 80, Bezons Cedex, France \\ ${ }^{d}$ Department of Materials Science, University of Milano-Bicocca, \\ Via Roberto Cozzi 55, Milano, Italy \\ E-mail: gianmaria.greco@poiesis.it, alexandra.nothnageldatos.net, \\ carlo.antonini@unimib.it
}

Over the past few years, the investigation of discrimination against students and researchers based on disability has become increasingly prominent, and it has broadened into a whole series of new perspectives and implications. Within this context, organisations, institutions and individuals have been promoting several actions for contrasting discriminatory bias and fostering a more inclusive environment for researchers and students with disabilities. One of the initiatives born in the wake of this movement is ResearchAbility, a multi-association initiative launched in 2018 that has become a subgroup of the Marie Curie Alumni Association since 2019. The main mission of ResearchAbility is twofold: (a) to support the careers of students and researchers with disabilities and (b) to promote research and awareness on disability and inclusive culture in the academic environment. After introducing the general context and mentioning data about students and staff with disabilities in UK academic institutions, the text introduces the ResearchAbility initiative, and describes some of its past activities and future areas of action.

The Ninth Annual Conference on Large Hadron Collider Physics - LHCP2021

7-12 June 2021

Online 


\section{Introduction}

While questions of social identity and discrimination within academia have been topics of discussion for decades, attention has increased considerably over the past few years. Scholars have been investigating this issue more and more, and calling for attention to be paid, for instance, to how gender and racial biases are embedded within the research environment and research culture $[1,2]$. Similarly, the question of disability in academia has been at the centre of debate among scholars, policy-makers and institutions for decades [3-5]. Over the past few years, the investigation of discrimination of students and researchers based on disability has become even more prominent, embracing a whole series of new perspectives [6-8]. The reason for such a significant change can be partially traced back to the adoption of the Convention on the Rights of Persons with Disabilities by the United Nations in 2007 [9]. The Convention was the fastest negotiated human rights treaty, with the highest number of signatories to a UN Convention on its opening day, and the first human rights treaty signed by the European Union as a legal party.

The Convention has been a game-changer on many fronts. One of its most noticeable effects has been the mainstreaming of the social model of disability. The two most prominent proposals for the interpretation of disability are the medical model of disability and the social model of disability [10]. According to the medical model, disability is caused by the condition of impairment of an individual. The impairment makes the individual deviate from some established normality defined by the dominant group. Within this model, actions usually focus on fixing individuals so as to make them comply, as close as possible, with the established condition of normality. Conversely, according to the social model, disability relates to a reciprocal process between individuals with impairments and an ableist society. From the perspective of the social model of disability, it is society that should be fixed, not individuals with impairments. Since it was first developed in the 1970s, the social model of disability has fostered the development of novel perspectives in the analysis of access problems and the devising of accessibility solutions $[11,12]$. The novel perspectives are also evident in the many recent analyses of discrimination of students and researchers with disabilities, which help to highlight the many forms of critical avoidance of disability in academia $[12,13]$.

Within this framework, the past few years have witnessed the development of several actions by organisations, institutions and individuals. One of the initiatives born in the wake of this newfound awareness is ResearchAbility (RA). Before describing the RA initiative however, some recent statistical data about students and staff with disabilities in UK academic institutions will help to sketch out the current situation within which RA operates.

\section{Researchers with disabilities: Some data from the UK}

A major problem in the assessment of the status of disability-based discrimination within research environments as well as the identification of the issues faced by researchers and students with disabilities is related to a lack of data. To date, there is not one comprehensive, detailed study on this topic at the European level, and the Eurostat data available is too limited to provide any solid ground for a systematic analysis. A similar situation can be found at most national levels. A recent exception is a survey carried out by Jisc on behalf of the Royal Society and published in January 2021, entitled Disability STEM data for students and academic staff in higher education 2007/08 to 2018/19 [14]. The document reports on a data analysis of (a) UK academic staff and 
(b) UK domiciled students, graduates and leavers in Science, Technology, Engineering and Mathematics (STEM) subjects. The data covers a period of twelve academic years - from 2007/08 to 2018/19 - and are based on the HESA Student and Staff records.

According to the report, the percentage of STEM entrants with a known disability increased consistently each year at both the first degree and postgraduate levels. Overall, the percentage doubled, increasing from $7.5 \%$ in $2007 / 08$ to $15.5 \%$ in $2018 / 19$ at the first degree level, and from $6.1 \%$ in $2007 / 08$ to $12.5 \%$ in $2018 / 19$ at the postgraduate level. The increase is mainly connected to a rise in student reporting of a specific learning difficulty or mental health condition. In terms of non-completion rate, there is little difference between first degree students with a known disability and those without; the difference is more significant however for postgraduate STEM students. The percentage of STEM students with a known disability who obtain first or upper second-class honours is lower than that of STEM students with no known disability. Finally, in the case of STEM leavers, the percentage of those with a known disability who are unemployed six months after graduation is higher than those with no known disability.

As for academic staff, the report highlights that the percentage of STEM academic staff with a known disability is rising year by year. More specifically, it has almost doubled, climbing from $2.0 \%$ in the $2007 / 08$ academic year to $3.8 \%$ in the 2018/19 academic year. Useful insights can be gained from data concerning conditions of employment. According to the report, "STEM academic staff with a known disability are more likely to have a contract that is teaching only compared to STEM academic staff with no known disability. [...] STEM academic staff with a known disability are less represented in more senior contract levels. They are less likely to hold a senior position than STEM academic staff with no known disability" (p. 5). While the data discussed in the report is limited only to UK academia, it is helpful to identify some of the major problems that students and researchers with disabilities may face along their path. It is precisely within such a challenging context that the RA initiative was born.

\section{The ResearchAbility initiative}

RA was established in 2018 as a multi-association initiative by a group of researchers following an initial proposal by one of the co-authors of this text. Since 2019, RA has been a subgroup of the Diversity \& Inclusivity Task Force within the Genders, Equity, Diversity and Inclusion Working Group of the Marie Curie Alumni Association (MCAA). The MCAA is an international non-profit organisation established and supported by the European Commission. It gathers together scholars from all career stages and scientific disciplines who benefit or have benefitted from funding within one of the Marie Skłodowska-Curie Actions (MSCA). The MCAA benefits from a vast international network of researchers, companies, and associations. Most notably, RA benefits from the mutual interaction between the MCAA and La fédé 100\% Handinamique, a French youth organisation that aims to reinforce the training and professional inclusion of young people with disabilities.

The main mission of RA is twofold: (a) to support the careers of students and researchers with disabilities and (b) to promote research and awareness on disability and inclusive culture in the academic environment. Strictly connected to its mission is the promotion of actions aimed at making the research environment more accessible. The RA initiative is grounded upon four pillars: (1) providing individual support; (2) educating the research ecosystem; (3) promoting research and expertise; and (4) fostering networking and policy-making. Jointly combined, the 
four pillars constitute the foundation of the overall action of the RA initiative: the promotion of an inclusive research system able to provide equitable chances for persons with disabilities. The main guiding principle of RA is expressed by the famous formula, "nothing about us without us." Within this social and pragmatic perspective, RA has been focussed on promoting several actions framed within one of the pillars or, more often, where two or more pillars intersect. One of the most notable actions carried out by RA is connected to the Marie Skłodowska-Curie Actions funding programme. In 2019, the MSCA programme was integrated with the introduction of a Special Needs Allowance (SNA). It is a separate funding programme for MSCA grant holders that aims to cover the high mobility costs (up to 60.000 EUR) faced by students, researchers and staff members with disabilities. RA provided support and feedback during the definition and evaluation of the SNA programme. In 2020, RA contributed to a campaign about the inclusion of a disability strategy in the French multi-year research programming law [15]. Other actions carried out by RA have focussed on the organisation of workshops, panels, and publications aimed at increasing awareness, the provision of support and the active search for funding, and the promotion of collaborations for and visibility of research about accessibility solutions, services, and policies.

\section{Future actions}

Students and researchers with disabilities face a multifaceted world of discrimination: not only in society but also within their professional environment. As shown by the many successful actions accomplished by RA in only its first few years of life, initiatives of this kind could provide significant support and encourage social change. However, the road towards an inclusive research environment is still long and ridden with obstacles. The past few years have witnessed a flood of national and international legislation related to disability and accessibility, as exemplified by the several regulations recently issued by the European Commission [16]. Despite this, the situation is still problematic. A case is point is the lack of homogeneity of national legal frameworks, evident in European countries. In relation to disability recognition, accommodations and social support, they can be divided into three main groups: countries that adopt a non-discrimination model with common law plans; countries that adopt a social protection model with quotas and a protected work sector; and countries that follow a mix of the two models. A major consequence is that the legal recognition of disability is not easily - or not at all - transferrable from one European country to another, with dire consequences for the individual's well-being and working conditions. Promoting the development of mechanisms that could allow for the transferability of such norms among European countries is one of RA's main areas of future action. A second focus of future action is that of lobbying for the inclusion of the SNA within Horizon Europe (HE) and other European research funding framework. While still available to people holding a grant or working within an MSCA project funded through the previous framework, i.e. Horizon 2020, the SNA is currently not included in HE. According to the current status of the HE framework, researchers and academic staff that will be involved in MSCA projects funded from 2021 onwards will not benefit from the support offered by this specific instrument. Further priorities for future actions include: the promotion of education programmes about accessibility, inclusion and diversity management; policy-making at European and national levels; actions for supporting the self-determination and self-identification of students and researchers with disabilities; and fostering the adoption of access services, in those that could support international mobility, and of disability-inclusive policies and strategies in education and research ecosystems. 


\section{References}

[1] J. Arday, "Fighting the tide: Understanding the difficulties facing Black, Asian and Minority Ethnic (BAME) Doctoral Students' pursuing a career in Academia," Educational Philosophy and Theory, vol. 53, no. 10, pp. 972-979, 2021, doi: 10.1080/00131857.2020.1777640.

[2] A. Llorens et al., "Gender bias in academia: A lifetime problem that needs solutions," Neuron, vol. 109, no. 13, pp. 2047-2074, 2021, doi: 10.1016/j.neuron.2021.06.002.

[3] A. Hurst, "Obstacles to overcome: higher education and disabled students.," in Uneasy Transitions: Disaffection in Post-Compulsory Education and Training, J. Corbett, Ed., London: Routledge, 1990, pp. 111-134.

[4] M. Nye, "The Higher Education Statistics Agency's data on students with disabilities in higher education," The Skill Journal, vol. 59, pp. 10-13, 1997.

[5] L. Lewis and E. Farris, An institutional perspective on students with disabilities in postsecondary education: (Report 6 of the National Committee of Inquiry into Higher Education). Washington DC: US Department of Education, National Center for Education Statistics, 1999.

[6] S.-D. Stone, V. A. Crooks, and M. Owen, "Going through the back door: Chronically ill academics' experiences as 'unexpected workers'," Soc Theory Health, vol. 11, no. 2, pp. 151-174, 2013, doi: 10.1057/sth.2013.1.

[7] J. Olsen, M. Griffiths, A. Soorenian, and R. Porter, "Reporting from the Margins: Disabled Academics Reflections on Higher Education," Scandinavian Journal of Disability Research, vol. 22, no. 1, pp. 265-274, 2020, doi: 10.16993/sjdr.670.

[8] N. Brown and J. Leigh, "Ableism in academia: where are the disabled and ill academics?," Disability \& Society, vol. 33, no. 6, pp. 985-989, 2018, doi: 10.1080/09687599.2018.1455627.

[9] G. M. Greco, "On accessibility as a human right, with an application to media accessibility," in Researching audio description: New approaches, A. Matamala and P. Orero, Eds., London: Palgrave Macmillan UK, 2016, pp. 11-33, doi: 10.1057/978-1-137-56917-2_2.

[10] D. Wasserman, A. Asch, J. Blustein, and D. Putnam, Disability: Definitions, Models, Experience. [Online]. Available: https://plato.stanford.edu/archives/sum2016/entries/ disability/

[11] G. M. Greco, "The nature of accessibility studies," Journal of Audiovisual Translation, vol. 1, no. 1, pp. 205-232, 2018, doi: 10.47476/jat.v1i1.51.

[12] G. M. Greco, "Towards a pedagogy of accessibility. The need for critical learning spaces in media accessibility education and training," Linguistica Antverpiensia, New Series: Themes in Translation Studies, vol. 18, pp. 23-46, 2019, doi: 10.52034/lanstts.v18i0.518.

[13] D. Bolt, "Social Encounters, Cultural Representation and Critical Avoidance," in Routledge handbook of disability studies, N. Watson, A. Roulstone, and C. Thomas, Eds., New York: Routledge, 2012.

[14] Jisc, Disability STEM data for students and academic staff in higher education 2007/08 to 2018/19. London: Jisc.

[15] M. Beuve, B. Blossier, H. Delacroix, S. Nashed, A. Nothnagel, L. Petitdemange, G. Ramstein, and P. E. Robert, "Ne pas oublier les personnels handicapés dans la loi programmation recherche", Le Monde, 2020. [Online]. Available : https://www.lemonde.fr/idees/article/2020/09/22/ne-pas-oublier-les-personnelshandicapes-dans-la-loi-programmation-recherche_6053190_3232.html

[16] G. M. Greco, "Fostering unity in diversity: The social value of European research in media accessibility," EuroScientist, 2019. [Online]. Available: https://www.euroscientist.com/ fostering-unity-in-diversity-the-social-value-of-european-research-on-media-accessibility/ 